\title{
Molecular Dynamics Simulation of SIRT1 Inhibitor from Indonesian Herbal Database
}

\author{
Andika ${ }^{1}$, Linda Erlina ${ }^{1}$, Azminah ${ }^{1}$, Arry Yanuar ${ }^{1}$ \\ 'Faculty of Pharmacy, Universitas Indonesia, Depok, West Java, INDONESIA.
}

\begin{abstract}
Objective: Sirtuins are protein deacetylases regulating cellular metabolism, lifespan, stress responses, and linked with diseases pathogenesis such as cancer and neurodegenerative diseases. SIRT1, one of human seven sirtuins, the most widely studied today. Hence, identification of SIRT1 drug compound has attracted in drug discovery community. To find good drug candidates could use in insilico methods as a quick tool for analyzing the biological activity of drugs virtually. Method: In silico methods in this research using molecular dynamics simulations that use Indonesia herbal database to identification hits compounds as the SIRT1 inhibitor. Analysis of molecular dynamics simulations in this study includes RMSD (root mean square deviation), RMSF (root mean square fluctuation), molecular mechanism Poisson-Boltzmann/surface area (MMPBSA) and hydrogen bonding. Results: The results showed that hits compounds, dregamine and 5-oxocoronaridine against two of macromolecules SIRT1 (PDB ID: 4I5I and 4ZZI) obtained free energy MMPBSA calculation about $-23 \mathrm{kcal} / \mathrm{mol}$ Meanwhile
\end{abstract}

occupancy hydrogen bonding of residues Ile347 and Asp348 about 80\% Conclusion: Hits compounds dregamine and 5-oxocoronaridine against two of macromolecules SIRT1 inhibitor (PDB ID: 4I5I and 4ZZI) obtained free energy MMPBSA calculation about $-23 \mathrm{kcal} / \mathrm{mol}$ meanwhile occupancy hydrogen bonding of residues Ile347 and Asp348 about $80 \%$.

Key Words: Sirtuin, SIRT1, Pharmacophore, Herbaldb, Molecular Dynamics Simulations.

\section{Correspondence:}

Arry Yanuar, Faculty of Pharmacy, Universitas Indonesia, Depok, INDONESIA.

Phone: +62-21-7270031

Email: arry.yanuar@ui.ac.id

DOI: 10.5530/jyp.2018.10.2

\section{INTRODUCTION}

Molecular dynamics (MD) simulations method is one of the first of pioneering applications for fluid dynamics by Alder and Wainwright and by Rahman in the late 1950s and early 1960s. Because of the revolutionary advances in computer technology and algorithmic improvements, MD then be a valuable tool in many fields of physics and chemistry. Since the 1970s the MD has been widely used to study the structure and dynamics of macromolecules, such as proteins or nucleic acids. ${ }^{1}$ Sirtuins are a nicotinamide adenine dinucleotide $\left(\mathrm{NAD}^{+}\right)$-dependent histone deacetylase with highly conserved from bacteria to mammals and have been showed to extend lifespan in yeast.,3 In mammals, there are 7 members (SIRT1-SIRT7) in the sirtuin family, which belong to class III histone deacetylases (HDACs) and show different functions, structure, and localization. ${ }^{4}$ Sirtuin1 (SIRT1), one of seven sirtuins members, has been extensively studied and evidently would be implicated in metabolism, senescence, and cancer. ${ }^{5}$ The NAD+-dependent deacetylase, has been described in the literature as a major player in the regulation of cellular stress responses. Its expression has been shown to be altered in cancer cells, and it targets both histone and non-histone proteins for deacetylation and thereby alters metabolic programs in response to diverse physiological stress. Interestingly, many of the metabolic pathways that are influenced by SIRT1 are also altered in tumor development. Not only does SIRT1 have the potential to regulate oncogenic factors, it also orchestrates many aspects of metabolism and lipid regulation and recent reports are beginning to connect these areas. SIRT1 influences pathways that provide an alternative means of deriving energy (such as fatty acid oxidation and gluconeogenesis, ${ }^{6,7}$ In addition, SIRT1 also involved in some neurodegenerative diseases, such as Huntington's and Alzheimer diseases. ${ }^{8,9}$ SIRT1 characterized by a conserved
275 amino acid catalytic core and unique additional N-terminal and/or C-terminal sequences of variable length. ${ }^{4}$

\section{MATERIALS AND METHODS}

\section{Materials}

SIRT1 is one of important drug target in drug discovery field. Threedimensional (3D) crystal structures for SIRT1 protein was obtained from the Protein Data Bank, PDB ID: 4I5I ${ }^{10}$ and PDB ID: 4ZZI. ${ }^{11}$

\section{Methods}

Molecular dynamics simulations using the AMBER $12^{12}$ and ff99SB force fields. Ligand parameters obtained from the general AMBER force field GAFF. ${ }^{13}$ Preparation of protein-ligand complexes, in addition to the counter ion, solvation, and preparation parameter/topology and coordinate files made using tLEaP module in AMBER. Four hits compound SIRT1 selected to proceed to the molecular dynamics simulations. Positive control compound selected in MD simulation is a ligand co-crystal and salermide. Partial atomic charge is calculated by the AM1BCC method that uses Antechamber software. The system was solvated using the water model TIP3BOX.14 Then all of the complex dissolved in water (using a water model TIP3PBOX) and cut boundary conditions octahedral periodic been applied (a cutoff distance of $10 \AA$ ). The entire systems were neutralized by adding $\mathrm{Na}^{+}$counter ions by replacing solvent molecules. The systems were subjected to 10,000 steps steepest descent energy minimization. The production run was $10 \mathrm{~ns}$. All simulations were run under periodic boundary conditions at the temperature $(300 \mathrm{~K})$ and the pressure constant (1 bar). Analysis of molecular dynamics simulations covers the RMSD (Root mean square deviation), RMSF 
(Root Mean Square Fluctuation), MMGBSA/MMPBSA and hydrogen bonding.

\section{RESULTS}

On the RMSD simulations, which lasted for 950 frames, each system has increased RMSD backbone indicating that the macromolecules unfold. There is an increase of four inhibitor compounds varied after frame 375. Ligand co-crystal $4 \mathrm{I} 5$ and hits compound shows RMSD value until a stable frame 950. 5-oxocoronaridine and dregamine increased to $4 \AA$ as well as salermide.

The value of RMSF outline illustrates the shift in the conformation of amino acid residues each that provide the flexibility of the protein. On RMSF against macromolecules PDB ID: 4I5I seen does not indicate high fluctuating during the simulation took place except on the last residue number. This indicates the residue is stable and does not provide the flexibility. Important residues such as Ala262, Phe273, Phe297, Ile314, Gln345, Asn346, Ile347, Asp348, and Val412 showed a low value of RMSF. This indicates that the residue is stable to bind to macromolecules and lacks flexibility (Figure 1). On RMSF backbone atoms against macromolecules PDB ID: 4ZZI at residue numbers 183-230 indicate fluctuating and high flexibility as a domain allosteric non-bonded ligand inhibitor compound (Figure 2).

Based on the docking scores and MMGBSA/MMPBSA binding free energies in Table 1, of the four compounds derived inhibitor hits compound 5-oxocoronaridine has the lowest free energy while salermide has the highest free energy between all four of these compounds. Meanwhile on the macromolecule PDB ID: 4ZZI dregamin hits compound has the lowest free energy while salermide has the highest free energy of the four of these compounds (Table 2).

Ligand co-crystal $4 \mathrm{I} 5$ have hydrogen bonding and binding affinity was good at residue Ile347 (94.21\%), Asp348 (66.53\%), Asn346 (63.16\%) and Gln345 (95.89\%). 5-oxocoronaridine showed hydrogen bonding interactions and binding affinity was good in Ile347 and Phe297 with occupancy respectively $87.37 \%$ and $50 \%$. Dregamin showed hydrogen bonding interactions and binding affinity on Ile316 with occupancy $73.05 \%$. The active compound in SIRT1, salermide have hydrogen bonding and good affinity at Glu315 with occupancy $69.89 \%$ (Table 3).

On the PDB ID: 4ZZI co-crystal 1NS form hydrogen bonds with residues Phe273, Asn346, Ile347, Asp348 and Val412 with value occupancy respectively $71.05 \%$; 54.32\%; 99.89\%; 96.11\%; 77.68\%. 5-oxocoronari-
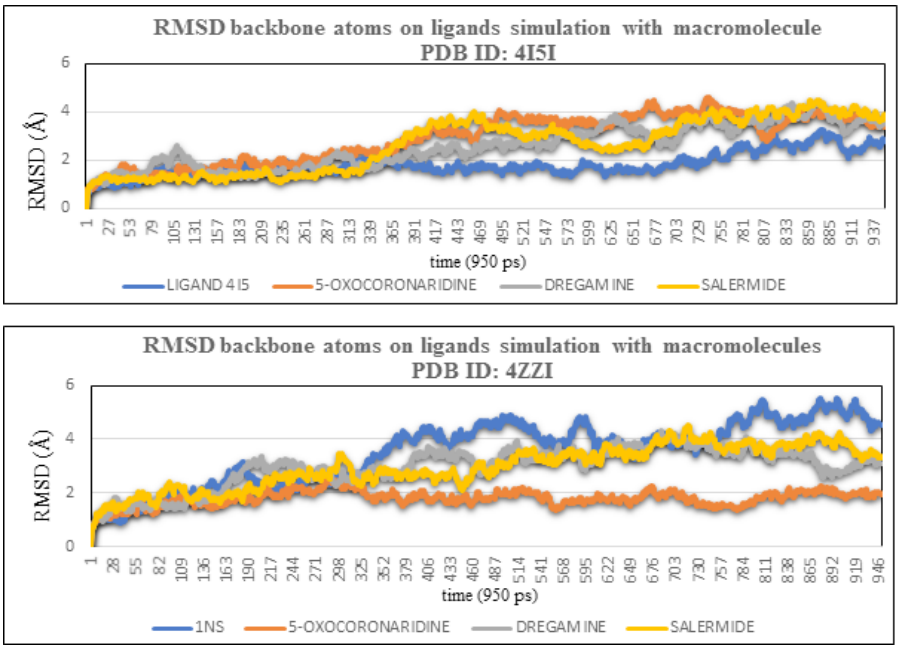

Figure 1: RMSD backbone atoms on ligands simulation with SIRT1 Macromolecules PDB ID: 4151 and 4ZZI.
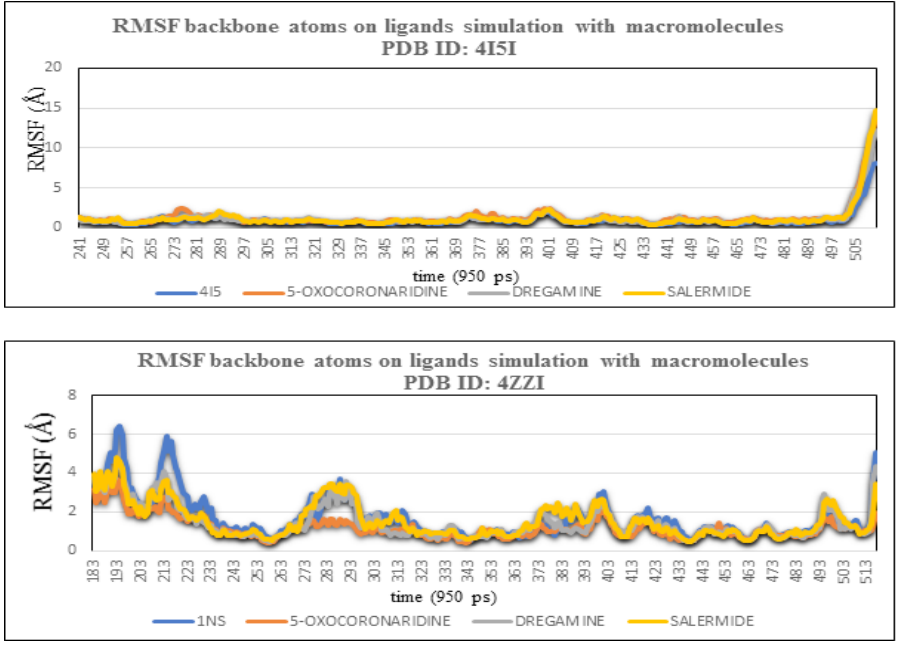

Figure 2: RMSF backbone atoms on ligands simulation with SIRT1 Macromolecules PDB ID: 4I5I and 4ZZI.

\begin{tabular}{cccc}
\multicolumn{4}{c}{ Table 1: Value Free Energy MMGBSA, MMPBSA and docking scores ligand / hits against SIRT1 macromolecule PDB ID: 4I5I } \\
\hline Compounds & DG MMGBSA $(\mathrm{kcal} / \mathrm{mol})$ & $\Delta \mathrm{G} \mathrm{MMPBSA}(\mathrm{kcal} / \mathrm{mol})$ & $\Delta$ G docking $(\mathrm{kcal} / \mathrm{mol})$ \\
\hline Ligand co-crystal 4i5 & -41.1278 & -30.6834 & -10.67 \\
Dregamine & -40.4032 & -25.7380 & -11.94 \\
5- oxocoronaridine & -45.1315 & -31.6695 & -10.05 \\
salermide & -38.8309 & -34.3201 & -12.10
\end{tabular}

\begin{tabular}{cccc} 
Table 2: Value Free Energy MMGBSA, MMPBSA and docking scores ligand / hits against SIRT1 macromolecule PDB ID: 4ZZI \\
\hline compound & \multicolumn{1}{c}{$\Delta \mathrm{G}$ MMGBSA (kcal / mol) } & $\begin{array}{c}\Delta \text { G MMPBSA } \\
\text { (kcal / mol) }\end{array}$ & \multicolumn{1}{c}{$\Delta$ G docking (kcal / mol) } \\
\hline Ligand co-crystal 1NS & -34.7746 & -20.0139 & -9.82 \\
Dregamine & -36.2641 & -22.8745 & -9.42 \\
5-oxocoronaridine & -30.0290 & -23.3249 & -8.93 \\
Salermide & -26.8162 & -21.2971 & -11.59 \\
\hline
\end{tabular}




\begin{tabular}{|c|c|c|c|}
\hline Ligand /Hits & Donor & Acceptor & Occupancy \\
\hline \multirow[t]{8}{*}{ Ligand co-crystal 4I5I (4I5) } & ILE347-Main-N-Side-O1 & $4 \mathrm{I} 5-515$ & $94.21 \%$ \\
\hline & ASP348-Main-N-Side-O1 & $4 \mathrm{I} 5-515$ & $66.53 \%$ \\
\hline & ILE347-Side-Side-CG1 & $4 \mathrm{I} 5275$ & $64.21 \%$ \\
\hline & 4I5275-O1-N1-Side-Side-OD1 & ASN346 & $63.16 \%$ \\
\hline & 4I5275-Side-N1 & ASP348-Side-OD2 & $94.84 \%$ \\
\hline & ASN346-CB-Side-Side-N1 & $4 \mathrm{I} 5275$ & $57.47 \%$ \\
\hline & 4I5275-N1-Side-Side-CB & ASN346 & $57.47 \%$ \\
\hline & 4I5275-Side-N2 & GLN345-Main-O & $95.89 \%$ \\
\hline \multirow[t]{2}{*}{ 5-oxocoronaridine } & ILE347-Main-N & LIG275- Side-O1 & $87.37 \%$ \\
\hline & PHE297-CZ-Side-Side-O2 & LIG275 & $50.00 \%$ \\
\hline Dregamine & ILE316-Side-CG2 & LIG275-Side-O1 & $73.05 \%$ \\
\hline Salermide & LIG275-Side-C3 & GLU315-Main-O & $69.89 \%$ \\
\hline
\end{tabular}

\begin{tabular}{cccc} 
Table 4: Inhibitor Compounds hydrogen bonding to the macromolecules PDB ID: 4ZZI & \multicolumn{1}{l}{ Occupancy } \\
\hline Ligand / Hits & Donor & Acceptor & $99.89 \%$ \\
\hline Ligand co-crystal 4ZZI (1NS) & ILE347 & -Main-N-Side-O171NS519 & $96.11 \%$ \\
& ASP348-Main-N-Side-O17 & 1 NS519 & $54.32 \%$ \\
& ASN346-Main-CA & 1NS519-Side-O17 & $71.05 \%$ \\
& PHE273-Main-N-Side-N19 & 1 NS519 & $77.68 \%$ \\
1NS519-Side-N03 & VAL412-Main-O & $99.89 \%$ \\
5-oxocoronaridine & INS519-Side-N16 & ASP348-Side-OD2 & $58.21 \%$ \\
& ILE347-Side-CG1 & 1NS519-Side-O17 & $73.58 \%$ \\
ALA262-Main-N-Side-O1 & LIG519 & $95.16 \%$ \\
Lregamine & LIG519-Main-N & GLN345-Main-O & $68.84 \%$ \\
& LIG519-C18-Side-Side-OD1 & ASN346 & $99.89 \%$ \\
\hline
\end{tabular}

dine form hydrogen bonds with residues Ala262, Gln345 and Asn346 with value occupancy respectively $73.58 \%$; $95.16 \%$; $68.84 \%$. The residue Asn346, Ile347, Asp348 interacts with the hit compound dregamine with value occupancy respectively $82.42 \%$; $99.89 \% ; 93.16 \%$. As for the active compound salermide form hydrogen bonds with residues Val412 with occupancy $63.68 \%$ (Table 4 ).

\section{DISCUSSION}

Extensive studies of sirtuins began in early 2000 when Guarente and coworkers found that Sir2 prolongs the life of yeast and lower organisms such as worms. ${ }^{3}$ Although well documented to prolong the lifespan of yeast, worms, flies, and mammals, SIRT1 has recently been involved in the initiation and progression of many dangerous diseases such as cancer. ${ }^{15}$ Sirtuin inhibitors may also be useful as therapeutic agents because of SIRT1 regulation of cancer. This raises the possibility of inhibition of SIRT1 can suppress the proliferation of cancer cells. ${ }^{16}$ Several small molecule acts as sirtuin inhibitor that has potentially useful as therapeutic agent and has been characterized, including splitomicin and its analogs ${ }^{17}$ sirtinol,${ }^{18}$ tenovin,${ }^{19}$ salermide,${ }^{20}$ thiobarbiturate,${ }^{21}$ cambinol, ${ }^{19}$ 2 -anilinobenzamide ${ }^{22}$ and other compounds. . $^{23,24,25}$

Simulation of molecular dynamics carried out by Karaman and Sippl (2015) by calculating MMGBSA with thieno core molecules [3,2-d] pyrimidine-6-carboxamide to SIRT1 shows all hydrogen bond interactions between the thieno [3,2-d] pyrimidine- 6-carboxamide and residue Ile347, Asp348 as well as water molecules required for inhibitor activity. The region amino acids residue Thr344 - Asp348 referred to as "floor" of the binding pocket possibility ligand binding for interaction site. Ile347 and Ile348 were extensively residue domain to binding pocket for the ligand. ${ }^{26}$ The results of the hydrogen bond analysis also show that residual Ile347 and Asp348 have high occupancy that plays a role in inhibitor activity. Based on research results the 5-oxocoronaridine and dregamine compounds are potential candidates for SIRT1 inhibitors.

\section{CONCLUSION}

Hits compounds dregamine and 5-oxocoronaridine against two of macromolecules SIRT1 inhibitor (PDB ID: 4I5I and 4ZZI) obtained free 
energy MMPBSA calculation about $-23 \mathrm{kcal} / \mathrm{mol}$ meanwhile occupancy hydrogen bonding of residues Ile347 and Asp348 about 80\%.

\section{ACKNOWLEDGEMENT}

Author (AY) thanks to the Publikasi Internasional Terindeks Untuk Tugas Akhir Mahasiswa (PITTA) 2016 grant from the Universitas Indonesia, for funding support.

\section{CONFLICT OF INTEREST}

The authors declare no conflict of interest.

\section{REFERENCES}

1. Karplus M, McCammon JA. Molecular dynamics simulations of biomolecules. Nat Struct Biol. 2002;9(9):646-52. Available from: http://www.nature.com/ doifinder/10.1038/nsb0902-646

2. Imai S, Armstrong CM, Kaeberlein M, Guarente L. Transcriptional silencing and longevity protein Sir2 is an NAD-dependent histone deacetylase. Nature. 2000:403(6771):795-800

3. Kaeberlein M, Kirkland KT, Fields S, Kennedy BK. Sir2-Independent Life Span Extension by Calorie Restriction in Yeast. PLoS Biol. 2004;2(9):1381-7.

4. Frye R a. Phylogenetic classification of prokaryotic and eukaryotic Sir2-like proteins. Biochem Biophys Res Commun. 2000 Jul 5 [cited 2015 Apr 11];273(2):793-8. Available from: http://www.ncbi.nlm.nih.gov/ pubmed/10873683

5. Jr GES, Pruitt WM, Pruitt K. Diverse Roles of SIRT1 in Cancer Biology and Lipid Metabolism. 2015;950-65.

6. Ota $H$, Tokunaga $E$, Chang $K$, Hikasa $M$, lijima $K$, Eto $M$, et al. Sirt1 inhibitor Sirtinol, induces senescence-like growth arrest with attenuated Ras-MAPK signaling in human cancer cells. Oncogene. 2006;25(2):176-85.

7. Portmann S, Fahrner R, Lechleiter A, Keogh A, Overney S, Laemmle A, et al. Antitumor Effect of SIRT1 Inhibition in Human HCCTumor Models in vitro and in vivo. Mol Cancer Ther. 2013;12(4):499-508. Available from: http://mct.aacrjournals.org/cgi/doi/10.1158/1535-7163.MCT-12-0700

8. Smith MR, Syed A, Lukacsovich T, Purcell J, Barbaro BA, Worthge SA, et al. A potent and selective sirtuin 1 inhibitor alleviates pathology in multiple animal and cell models of huntington's disease. Hum Mol Genet. 2014;23(11):2995-3007.

9. Albani D, Polito L, Forloni G. Sirtuins as novel targets for Alzheimer's disease and other neurodegenerative disorders: Experimental and genetic evidence. J Alzheimer's Dis. 2010;19(1):11-26.

10. Davenport AM, Huber FM, Hoelz A. Structural and Functional Analysis of Human SIRT1. J Mol Biol. The Authors; 2014;426(3):526-41. Available from: http://dx.doi.org/10.1016/j.jmb.2013.10.009

11. Dai H, Case AW, Riera TV, Considine T, Lee JE, HamuroY, et al. Crystallographic structure of a small molecule SIRT1 activator-enzyme complex. Nat Commun. 2015;6:7645. Available from: http://www.nature.com/doifinder/10.1038/ncomms8645

12. Case DA, Darden T, Cheatham TE, Simmerling C, Wang J, Duke RE, et al. Amber 12. Univ California, San Fr. 2012;
13. Wang J, Wolf RM, Caldwell JW, Kollman PA, Case DA. Development and testing of a general amber force field. J Comput Chem. 2004;25(9):1157-74. Available from: http://doi.wiley.com/10.1002/jcc.20035

14. Jorgensen WL, Chandrasekhar J, Madura JD, Impey RW, Klein ML. Comparison of simple potential functions for simulating liquid water. J Chem Phys. 1983;79(2):926. Available from: http://scitation.aip.org/content/aip/journal/ jcp/79/2/10.1063/1.445869

15. Liu T, Liu PY, Marshall GM. The Critical Role of the Class III Histone Deacetylase SIRT1 in Cancer. Cancer Res. 2009;69(5):1702-5. Available from: http:// cancerres.aacrjournals.org/cgi/doi/10.1158/0008-5472.CAN-08-3365

16. Kozako T, Suzuki T, Yoshimitsu M, Arima N, Honda S, Soeda S. Anticancer Agents Targeted to Sirtuins. Molecules. 2014;19(12):20295-313. Available from: http://www.mdpi.com/1420-3049/19/12/20295/

17. Posakony J, Hirao M, Stevens S, Simon JA, Bedalov A. Inhibitors of Sir2: Evaluation of Splitomicin Analogues. J Med Chem. 2004;47(10):2635-44. Available from: http://pubs.acs.org/doi/abs/10.1021/jm030473r

18. Grozinger CM, Chao ED, Blackwell HE, Moazed D, Schreiber SL. Identification of a Class of Small Molecule Inhibitors of the Sirtuin Family of NAD-dependent Deacetylases by Phenotypic Screening. J Biol Chem. 2001;276(42):38837-43.

19. Lain S, Hollick JJ, Campbell J, Staples OD, Higgins M, Aoubala M, et al. Discovery, In Vivo Activity, and Mechanism of Action of a Small-Molecule p53 Activator Cancer Cell. 2008;13(5):454-63.

20. Lara E, Mai A, Calvanese V, Altucci L, Lopez-Nieva P, Martinez-Chantar ML, et al. Salermide, a Sirtuin inhibitor with a strong cancer-specific proapoptotic effect. Oncogene. 2009;28(6):781-91. Available from: http://www.nature.com/ doifinder/10.1038/onc.2008.436

21. Uciechowska U, Schemies J, Neugebauer RC, Huda E, Schmitt ML, Meier R, et al. Thiobarbiturates as Sirtuin Inhibitors: Virtual Screening, Free-Energy Calculations, and Biological Testing. Chem Med Chem. 2008;3(12):1965-76. Available from: http://doi.wiley.com/10.1002/cmdc.200800104

22. Suzuki T, Imai K, Imai E, lida S, Ueda R, Tsumoto $H$, et al. Design, synthesis, enzyme inhibition, and tumor cell growth inhibition of 2-anilinobenzamide derivatives as SIRT1 inhibitors. Bioorg Med Chem. Elsevier Ltd: 2009;17(16):5900-5. Available from: http://dx.doi.org/10.1016/j.bmc.2009.07.001

23. Kim H, Kim M, Lee S, Lee J, Bae J, Kim D, et al. A novel SIRT1 inhibitor, sensitizes TRAIL-resistant human leukemic K562 cells to TRAIL-induced apoptosis. Biochem Pharmacol. Elsevier Inc.; 2012;84(3):402-10. Available from: http://dx.doi. org/10.1016/j.bcp.2012.03.014

24. Kalle AM, Mallika A, Badiger J, Alinakhi, Talukdar P, Sachchidanand. Inhibition of SIRT1 by a small molecule induces apoptosis in breast cancer cells. Biochem Biophys Res Commun. Elsevier Inc.; 2010;401(1):13-9. Available from: http:// dx.doi.org/10.1016/j.bbrc.2010.08.118

25. Wu J, Li Y, Chen $\mathrm{K}$, Jiang $H, X u M-H$, Liu D. Identification of benzofuran3-yl(phenyl)methanones as novel SIRT1 inhibitors: Binding mode, inhibitory mechanism and biological action. Eur J Med Chem. 2013;60:441-50. Available from: http://linkinghub.elsevier.com/retrieve/pii/S0223523412007489

26. Huhtiniemi T, Wittekindt C, Laitinen T, Leppänen J, Salminen A, Poso A, et al. Comparative and pharmacophore model for deacetylase SIRT1. J Comput Aided Mol Des. 2006;20(9):589-99. Available from: http://link.springer.com/10.1007/ s10822-006-9084-9

Article History: Submission Date : 24-08-2017 ; Revised Date : 07-10-2017; Acceptance Date : 25-11-2017.

Cite this article: Andika, Erlina L, Azminah and Yanuar A. Molecular Dynamics Simulation of SIRT1 Inhibitor from Indonesian Herbal Database. J Young Pharm. 2018;10(1):3-6 